\section{Efficient Decompression of Binary Encoded Balanced Ternary Sequences}

Olivier Muller, Adrien Prost-Boucle, Alban Bourge, Frédéric Pétrot Member, IEEE

\begin{abstract}
A balanced ternary digit, known as a trit, takes its values in $\{-1,0,1\}$. It can be encoded in binary as $\{11,00,01\}$ for direct use in digital circuits. In this correspondence, we study the decompression of a sequence of bits into a sequence of binary encoded balanced ternary digits. We first show that it is useless in practice to compress sequences of more than 5 ternary values. We then provide two mappings, one to map 5 bits to 3 trits and one to map 8 bits to 5 trits. Both mappings were obtained by human analysis and lead to boolean implementations that compare quite favorably with others obtained by tweaking assignment or encoding optimization tools. However, mappings that lead to better implementations may be feasible.
\end{abstract}

\section{INTRODUCTION}

Ternary encoding of data has been shown useful at least in the following contexts: general purpose computing [1], wireless transmission [2], [3], texture representation in images [4], quantum computing [5], optical super computing [6], and artificial neural networks [7][8].

In many applications, the binary value representing a sequence of $\{-1,0,1\}$ needs to be stored in memory, so finding an encoding that minimizes both the compressor and decompressor is a legitimate goal. However, our own focus is the VLSI implementation of neural networks making use of ternary weigths, in which the weight values are written in a memory only once and read almost continuously [9]. In that case, it is necessary to combinatorially produce a sequence of binary encoded balanced ternary values from an encoded binary string.

Our objective is thus to determine a mapping (i.e. a one-toone function that maps binary strings to binary encoded balanced ternary values) which, when implemented as a boolean multi-valued function, leads to factored-form expressions with the least number of boolean operators and the least number of literals (considering also the outputs of previous operators) as operands of those operators. This factored-form representation is interesting because it approximates the complexity of a gate-level implementation [10]. The only constraint we have is the encoding of the ternary values, given by $\mu:\{-1,0,1\} \mapsto\{11,00,01\}$. This choice is appropriate for use in classical two's complement arithmetic circuits, for instance when these values are directly fed into multipliers or adders [9].

In the following sections, we show that it is not useful to compress more than 5 trits on 8 bits, and give the best mappings that we found, i.e. the ones requiring fewer gates, for compressing 3 trits on 5 bits and 5 trits on 8 bits. Please note that we do not propose a general algorithm to solve the problem for sequences of any length.

\section{PROBLEM FORMULATION}

A ternary digit contains $\log _{2}(3) \approx 1.586$ bits of information. We compute the maximum theoretical gain that can be obtained by compressing trits in binary. As a sequence of $n$ trits $(n \in \mathbb{N})$ represents $3^{n}$ values, at least $\left\lceil\log _{2}\left(3^{n}\right)\right\rceil=\left\lceil n \log _{2}(3)\right\rceil$ bits are necessary to

O. Muller and F. Pétrot are with Univ. Grenoble Alpes, Grenoble-INP (Institute of Engineering Univ. Grenoble Alpes), TIMA, F-38000 Grenoble, France (e-mail: \{olivier.muller,frederic.petrot\}@univ-grenoble-alpes.fr).

A. Prost-Boucle was Univ. Grenoble Alpes, TIMA, F-38000 Grenoble, France. He is now with Synopsys, 38330 Montbonnot-Saint-Martin, France (e-mail: adrien.prost-boucle@ synopsys.com).

A. Bourge was Univ. Grenoble Alpes, TIMA, F-38000 Grenoble, France. He is now with Atos, 38130 Échirolles, France (e-mail: alban.bourge@ atos.net).
TABLE I: Gain and free values for encoding trits on bits

\begin{tabular}{|r|r|r|r|}
\hline trits & bits & gain & $\begin{array}{r}\text { free values } \\
\left(2^{\text {bits }}-3^{\text {trits }}\right)\end{array}$ \\
\hline 1 & 2 & $0.00 \%$ & 1 \\
\hline 2 & 4 & $0.00 \%$ & 7 \\
\hline 3 & 5 & $16.67 \%$ & 5 \\
\hline 4 & 7 & $12.50 \%$ & 47 \\
\hline 5 & 8 & $20.00 \%$ & 13 \\
\hline 6 & 10 & $16.67 \%$ & 295 \\
\hline 7 & 12 & $14.29 \%$ & 1909 \\
\hline 8 & 13 & $18.75 \%$ & 1631 \\
\hline 9 & 15 & $16.67 \%$ & 13085 \\
\hline 10 & 16 & $20.00 \%$ & 6487 \\
\hline \hline 17 & 27 & $20.58 \%$ & 5077565 \\
\hline
\end{tabular}

encode this sequence in binary. For $n$ trits the gain compared to the non encoded sequence is given by $u_{n}=\frac{2 n-\left\lceil n \log _{2}(3)\right\rceil}{2 n}$. By definition $x \leq\lceil x\rceil<x+1$, hence

$$
\underbrace{\frac{2 n-n \log _{2}(3)}{2 n}}_{v_{n}} \geq u_{n}>\underbrace{\frac{2 n-\left(n \log _{2}(3)+1\right)}{2 n}}_{w_{n}}
$$

Yet $\forall n \in \mathbb{N}, v_{n}=1-\frac{\log _{2}(3)}{2}$ and $\lim _{n \rightarrow \infty} w_{n}=1-\frac{\log _{2}(3)}{2}$. Since $v_{n}$ and $w_{n}$ increase monotonically, this yields by the squeeze theorem $\lim _{n \rightarrow \infty} u_{n}=1-\frac{\log _{2}(3)}{2} \approx 0.2075$.

Table I gives the actual gain for small values of $n$. As can be seen, there is not much interest in encoding sequences of more than 5 trits (actually 10 bits) on 8 bits, since it is at $\approx 0.75 \%$ of the maximum achievable gain. The next higher gain, obtained for 17 trits, is given in the table for completeness.

Given $b$ bits and $t$ trits, we have to determine how to map $2^{b}$ values onto $3^{t}$ values so that the multi-level logic implementation is minimized, i.e. leads to the use of as few boolean operators as possible with each of these operators having an as low number of inputs as possible. From a combinatorial point of view these mappings are ordered arrangements, so there are $2^{b \frac{3^{t}}{}}=\frac{2^{b} !}{\left(2^{b}-3^{t}\right)}$ of them, where $n^{\underline{m}}$ represents the falling factorial. We focus on two particular instances of the problem, the mapping of 3 trits on 5 bits, leading to $32^{\frac{27}{2}} \approx 2.210^{33}$ possible mappings, and the mapping of 5 trits on 8 bits, leading to $256^{\underline{243}} \approx 1.410^{497}$ mappings to choose from.

It is quite clear given this analysis that searching for an optimized mapping of 17 trits on 27 bits is totally unpractical.

Even for our two cases of relatively small size, given the size of the search space, exhaustive search is not an option, and finding the optimal solutions is statistically unlikely since multi-level optimization is an NP-complete problem [11].

\section{RELATED WORK}

This problem may seem a fairly well known one, but to our surprise, the work of mapping a bit string representing a subset of its possible values to a subset of bit strings of smaller size containing all permutations does not seem documented in the literature.

The most extensive surveys on logic synthesis and input/output encoding and state assignment [10], [12] target slightly different problems, making the available techniques not easily transposable. Another approach to obtain state assignment, by using symbolic representation of the states as proposed in [13], reaches optimal solutions with more than the minimal number of bits, whereas it is critical in our case to stick to the minimum number of bits. Chapter 7.5 of De Micheli's book [14] is dedicated to these encoding problems, but again, the problems that are solved are sufficiently different from ours to make the approaches inappropriate.

Output encoding is also the subject of [15]. This paper cites all the relevant works on the topic of encoding targeting logic minimization. 
We refer the interested reader to its bibliography, to avoid too many citations in this correspondence. This work makes the assumption that the mapping is already chosen, although part of the output uses symbols instead of bits, and it is the bit strings corresponding to these symbols that are searched for. Their proposition is also unfortunately not generalizable to our problem.

Bearing in mind that our goal is not to devise the best assignment for this problem in its generality, but for the two instances that we believe are useful, we focus on these problems only.

\section{EVALUATION APPROACH}

We take as first approximation of the logic complexity the number of literals after multi-level minimization and technology mapping on a minimal standard cell library using the Alliance VLSI CAD tools [16]. We also tried with Espresso [17] followed by Alliance technology mapping, but the results where not consistent with Synopsys synthesis as Espresso targets multi-level PLA minimization and not standard cells. The library contains exclusively a not and 2, 3 and 4-input and, or, nand, nor, xor, and xnor gates, and uses arbitrary units for area $(\lambda)$. This step allows first to rank the solutions easily and second to reproduce the results presented here, without the need to access a specific proprietary software and cell library.

As a first step, we generated 10,000 random assignments for the two interesting cases, using the mapping $\mu$ for the ternary values representation. The mapping of 32 values coded on 5 bits to the 27 legal 3 trits coded on 6 bits led to an average number of gates equal to $\approx 77.4$ with a standard deviation of $\approx 6.0$ after technology mapping. Regarding the assignment of 256 values coded on 8 bits to the 243 legal 5 trits coded on 10 bits, the average number of gates equals to $\approx 886.8$ with a standard deviation of $\approx 14.8$ after technology mapping. We give here the number of gates, but we verified that, given the limited number of gates in the standard cell library, there is a very strong correlation with the area.

As a second step, and for actual implementation, we use Synopsys' design compiler targeting STMicroelectronics $28 \mathrm{~nm}$ FDSOI standard cell library at an operating point of $0.9 \mathrm{~V}$. The areas and propagation times of the circuits are given as reported by the synthesis tool.

\section{ENCODING SOLUTIONS}

We tried several automated solutions that failed to minimize the number of gates. The Hungarian algorithm [18] is optimal for assignment problem, as long as we can provide a cost matrix. We are unable to build a relevant cost matrix. Indeed, costs are interdependent since boolean subexpressions are shared. We attempted unsuccessfully with several cost functions being variation of the Hamming distance between the trits code and the binary values. We also tried to tweak state assignment algorithms to perform this assignment instead of state assignment. Overall, these trials produce encodings that, once synthesized, contain half the number of gates of a random assignment, but are still far from the solution we present below (at least twice as big for the 8 bits to 5 trits case).

In this work, we did not consider classical algorithms used for large NP-complete problems such as simulated annealing, generic algorithms or tabu search. To perform efficiently, these algorithms need a fast and accurate evaluation of the solutions to deal with the huge number of produced solutions during searching. Unfortunately, a ASIC synthesis on the chosen pre-characterized library of logic cells requires at least a minute.

The solutions presented below where derived by hand assuming structural properties. Regarding the notation, '-' represents a "don't care", i.e. the value of the signal is irrelevant.

\section{A. 5 bits to 3 trits}

Denoting $t_{5 . .0}$ the binary encoded ternary values and $b_{4 . .0}$ the binary codes, our best assignment solution is:

\begin{tabular}{|l|l|}
\hline$t_{5} \ldots t_{0}$ & $b_{4} \ldots b_{0}$ \\
\hline 000000 & -0001 \\
\hline 000001 & -0010 \\
\hline 000011 & -0110 \\
\hline 000100 & $00-00$ \\
\hline 001100 & $01-00$ \\
\hline 010101 & 00011 \\
\hline 010100 & 00101 \\
\hline 010111 & 00111 \\
\hline 010000 & 01001 \\
\hline 010001 & 01010 \\
\hline 011101 & 01011 \\
\hline 011100 & 01101 \\
\hline 010011 & 01110 \\
\hline 011111 & 01111 \\
\hline
\end{tabular}

\begin{tabular}{|l|l|}
\hline$t_{5} \ldots t_{0}$ & $b_{4} \ldots b_{0}$ \\
\hline 000101 & 10000 \\
\hline 110101 & 10011 \\
\hline 000111 & 10100 \\
\hline 110100 & 10101 \\
\hline 110111 & 10111 \\
\hline 001101 & 11000 \\
\hline 110000 & 11001 \\
\hline 110001 & 11010 \\
\hline 111101 & 11011 \\
\hline 001111 & 11100 \\
\hline 111100 & 11101 \\
\hline 110011 & 11110 \\
\hline 111111 & 11111 \\
\hline & \multicolumn{2}{|l}{} \\
\hline
\end{tabular}

We now detail how we have obtained this solution. The principle is as follows. First, we generate each trit by coding only its magnitude $\left(t_{4}, t_{2}\right.$, or $\left.t_{0}\right)$, i.e. whether it is null or not. The sign is obtained thanks to one input bit only $\left(b_{4}, b_{3}\right.$, or $\left.b_{2}\right)$. The codes are then $\left(b_{4} . t_{4}, t_{4}, b_{3} . t_{2}, t_{2}, b_{2} . t_{2}, t_{2}\right)$. We call $\left(t_{4}, t_{2}, t_{0}\right)$ the magnitude vector. Second, the idea is to gather codes having similar magnitude vectors in sets. In our proposal, the first set contains the 8 codes associated to the magnitude vector $(1,1,1)$. The second set contains 6 codes, 4 associated to the magnitude vector $(0,1,1)$ and 2 associated to $(0,1,0)$. In this set, the most significant trit is 0 . Thus, $b_{4}$ can be reused and the magnitude vector can be $\left(0,1, b_{4}\right)$. Similarly, the third set contains the 6 codes corresponding to the magnitude vector $\left(b_{3}, 0,1\right)$. The last set contains the last 7 codes, 4 associated to the magnitude vector $(1,1,0), 2$ associated to $(1,0,0)$ and one to $(0,0,0)$. The first 6 codes can be efficiently expressed by magnitude vector $\left(1, b_{2}, 0\right)$. When $b_{2}$ is $0, b_{3}$ is unused. It is then used to distinguish $(1,0,0)$ from $(0,0,0)$. So we can extend the magnitude vector to $\left(b_{3}+b_{2}, b_{2}, 0\right)$ to cover all cases. These 4 sets are respectively encoded as ' 11 ', '00', ' 10 ' and ' 01 ' using $b_{1} b_{0}$.

From classical boolean optimization [19] and factorization techniques, we derive the following equations:

$$
\begin{aligned}
x_{0} & =b_{0}\left(b_{1}+b_{2}\right), x_{1}=b_{0}+b_{1} \\
t_{0} & =b_{1}+\overline{b_{0}} b_{4}, t_{1}=t_{0} b_{2} \\
t_{2} & =x_{0}+\overline{x_{1}}, t_{3}=t_{2} b_{3} \\
t_{4} & =x_{0}+b_{3} x_{1}, t_{5}=t_{4} b_{4}
\end{aligned}
$$

This solution can be synthesized in 17 gates with an area of $17250 \lambda^{2}$ with Alliance. Compared to the random generated cases, it is about 4.5 times smaller. Synthesis on STMicro $28 \mathrm{~nm}$ FDSOI using the entire standard cell library produces an area of $6.52 \mu \mathrm{m}^{2}$, (11 gates instantiated), and a propagation time of $41 \mathrm{ps}$.

\section{B. 8 bits to 5 trits}

Again, denoting $t_{9 . .0}$ the binary encoded ternary values and $b_{7 . .0}$ the binary codes, our best assignment solution is:

\begin{tabular}{|l|l|}
\hline$t_{9} \ldots t_{0}$ & $b_{7} \ldots b_{0}$ \\
\hline 0100010101 & 00000000 \\
\hline 0101000001 & 00000001 \\
\hline 0101000000 & 00000010 \\
\hline 0101010100 & 00000011 \\
\hline 0101000101 & 00000100 \\
\hline 0101010001 & 00000101 \\
\hline 0101010000 & 00000110 \\
\hline 0101010101 & 00000111 \\
\hline 0001010101 & 00001000 \\
\hline 0001000000 & $000-1001$ \\
\hline 0100010100 & 00001010 \\
\hline 0001010100 & 00001011 \\
\hline 0100000101 & 00001100 \\
\hline 0001000001 & 00001101 \\
\hline
\end{tabular}

\begin{tabular}{|c|c|}
\hline$t_{9} \ldots t_{0}$ & $b_{9} \ldots b_{0}$ \\
\hline 0111111111 & 01111111 \\
\hline 1100010101 & 10000000 \\
\hline 1101000001 & 10000001 \\
\hline 1101000000 & 10000010 \\
\hline 1101010100 & 10000011 \\
\hline 1101000101 & 10000100 \\
\hline 1101010001 & 10000101 \\
\hline 1101010000 & 10000110 \\
\hline 1101010101 & 10000111 \\
\hline 0011010101 & 10001000 \\
\hline 0011000000 & $100-1001$ \\
\hline 0000010101 & 10001010 \\
\hline 0001010001 & 10001011 \\
\hline 1100000101 & 10001100 \\
\hline
\end{tabular}




\begin{tabular}{|c|c|c|c|}
\hline \begin{tabular}{ll|}
$t_{9}$ & $\ldots$ \\
\end{tabular} & $b_{7}$ & $t_{9}$ & $\begin{array}{ll}\cdots & b_{0} \\
\end{array}$ \\
\hline 0000010100 & -0001110 & 0011000001 & 10001101 \\
\hline 0101010111 & 00001111 & 1101010111 & 10001111 \\
\hline 0100010111 & 00010000 & 1100010111 & 10010000 \\
\hline 0101000011 & 00010001 & 1101000011 & 10010001 \\
\hline 0100000100 & $0-010010$ & 1100000100 & $1-010010$ \\
\hline 0101011100 & 00010011 & 1101011100 & 10010011 \\
\hline 0101000111 & 00010100 & 1101000111 & 10010100 \\
\hline 0101010011 & 00010101 & 1101010011 & 10010101 \\
\hline 0101000100 & 00010110 & 1101000100 & 10010110 \\
\hline 0101011101 & 00010111 & 1101011101 & 10010111 \\
\hline 0001010111 & 00011000 & 0011010111 & 10011000 \\
\hline 1100010100 & 00011010 & 0000010111 & 10011010 \\
\hline 0001011100 & 00011011 & 0001010011 & 10011011 \\
\hline 0100000111 & 00011100 & 1100000111 & 10011100 \\
\hline 0001000011 & 00011101 & 0011000011 & 10011101 \\
\hline 0000011100 & -0011110 & 1101011111 & 10011111 \\
\hline 0101011111 & 00011111 & 1100011101 & 10100000 \\
\hline 0100011101 & 00100000 & 1100010001 & 10100001 \\
\hline 0100010001 & 00100001 & 1100010000 & 10100010 \\
\hline 0100010000 & 00100010 & 1101110100 & 10100011 \\
\hline 0101110100 & 00100011 & 1101001101 & 10100100 \\
\hline 0101001101 & 00100100 & 1101110001 & 10100101 \\
\hline 0101110001 & 00100101 & 1101110000 & 10100110 \\
\hline 0101110000 & 00100110 & 1101110101 & 10100111 \\
\hline 0101110101 & 00100111 & 0011011101 & 10101000 \\
\hline 0001011101 & 00101000 & 0000000001 & 10101001 \\
\hline 0100000000 & 00101001 & 0000011101 & 10101010 \\
\hline 0100011100 & 00101010 & 0001110001 & 10101011 \\
\hline 0001110100 & 00101011 & 1100001101 & 10101100 \\
\hline 0100001101 & 00101100 & 1100000001 & 10101101 \\
\hline 0100000001 & 00101101 & 1101110111 & 10101111 \\
\hline 0000110100 & -0101110 & 1100011111 & 10110000 \\
\hline 0101110111 & 00101111 & 1100010011 & 10110001 \\
\hline 0100011111 & 00110000 & 1100001100 & $1-110010$ \\
\hline 0100010011 & 00110001 & 1101111100 & 10110011 \\
\hline 0100001100 & $0-110010$ & 1101001111 & 10110100 \\
\hline 0101111100 & 00110011 & 1101110011 & 10110101 \\
\hline 0101001111 & 00110100 & 1101001100 & 10110110 \\
\hline 0101110011 & 00110101 & 1101111101 & 10110111 \\
\hline 0101001100 & 00110110 & 0011011111 & 10111000 \\
\hline 0101111101 & 00110111 & 0000000011 & 10111001 \\
\hline 0001011111 & 00111000 & 0000011111 & 10111010 \\
\hline 1100000000 & 00111001 & 0001110011 & 10111011 \\
\hline 1100011100 & 00111010 & 1100001111 & 10111100 \\
\hline 0001111100 & 00111011 & 1100000011 & 10111101 \\
\hline 0100001111 & 00111100 & 1101111111 & 10111111 \\
\hline 0100000011 & 00111101 & 1100110101 & 11000000 \\
\hline 0000111100 & -0111110 & 1111000001 & 11000001 \\
\hline 0101111111 & 00111111 & 1111000000 & 11000010 \\
\hline 0100110101 & 01000000 & 1111010100 & 11000011 \\
\hline 0111000001 & 01000001 & 1111000101 & 11000100 \\
\hline 0111000000 & 01000010 & 1111010001 & 11000101 \\
\hline 0111010100 & 01000011 & 1111010000 & 11000110 \\
\hline 0111000101 & 01000100 & 1111010101 & 11000111 \\
\hline 0111010001 & 01000101 & 0011110101 & 11001000 \\
\hline 0111010000 & 01000110 & 0000010000 & 11001001 \\
\hline 0111010101 & 01000111 & 0000110101 & 11001010 \\
\hline 0001110101 & 01001000 & 0011010001 & 11001011 \\
\hline 0000000100 & $010-1001$ & 0011000101 & 11001100 \\
\hline 0100110100 & 01001010 & 0000010001 & 11001101 \\
\hline 0011010100 & 01001011 & 0011010000 & 11001110 \\
\hline 0001000101 & 01001100 & 1111010111 & 11001111 \\
\hline 0000000101 & 01001101 & 1100110111 & 11010000 \\
\hline 0001010000 & 01001110 & 1111000011 & 11010001 \\
\hline 0111010111 & 01001111 & 1111011100 & 11010011 \\
\hline 0100110111 & 01010000 & 1111000111 & 11010100 \\
\hline 0111000011 & 01010001 & 1111010011 & 11010101 \\
\hline 0111011100 & 01010011 & 1111000100 & 11010110 \\
\hline 0111000111 & 01010100 & 1111011101 & 11010111 \\
\hline 0111010011 & 01010101 & 0011110111 & 11011000 \\
\hline 0111000100 & 01010110 & 0000000000 & $11-11001$ \\
\hline 0111011101 & 01010111 & 0000110111 & 11011010 \\
\hline 0001110111 & 01011000 & 0011010011 & 11011011 \\
\hline 1100110100 & 01011010 & 0011000111 & 11011100 \\
\hline 0011011100 & 01011011 & 0000010011 & 11011101 \\
\hline 0001000111 & 01011100 & 0011000100 & 11011110 \\
\hline 0000000111 & 01011101 & 1111011111 & 11011111 \\
\hline 0001000100 & 01011110 & 1100111101 & 11100000 \\
\hline 0111011111 & 01011111 & 1100110001 & 11100001 \\
\hline 0100111101 & 01100000 & 1100110000 & 11100010 \\
\hline 0100110001 & 01100001 & 1111110100 & 11100011 \\
\hline 0100110000 & 01100010 & 1111001101 & 11100100 \\
\hline 0111110100 & 01100011 & 1111110001 & 11100101 \\
\hline 0111001101 & 01100100 & 1111110000 & 11100110 \\
\hline 0111110001 & 01100101 & 1111110101 & 11100111 \\
\hline
\end{tabular}

\begin{tabular}{|c|c|c|c|}
\hline $\begin{array}{ll}\ldots & t_{0}\end{array}$ & $\begin{array}{lll}b_{7} & \ldots & b_{0}\end{array}$ & $\begin{array}{ll}t_{9} & \ldots \\
\end{array}$ & $\begin{array}{ll}. . & b_{0}\end{array}$ \\
\hline 0111110000 & 01100110 & 0011111101 & 11101000 \\
\hline 0111110101 & 01100111 & 0000110000 & 11101001 \\
\hline 0001111101 & 01101000 & 0000111101 & 11101010 \\
\hline 0000001100 & $011-1001$ & 0011110001 & 11101011 \\
\hline 0100111100 & 01101010 & 0011001101 & 11101100 \\
\hline 0011110100 & 01101011 & 0000110001 & 11101101 \\
\hline 0001001101 & 01101100 & 0011110000 & 11101110 \\
\hline 0000001101 & 01101101 & 1111110111 & 11101111 \\
\hline 0001110000 & 01101110 & 1100111111 & 11110000 \\
\hline 0111110111 & 01101111 & 1100110011 & 11110001 \\
\hline 0100111111 & 01110000 & 1111111100 & 11110011 \\
\hline 0100110011 & 01110001 & 1111001111 & 11110100 \\
\hline 0111111100 & 01110011 & 1111110011 & 11110101 \\
\hline 0111001111 & 01110100 & 1111001100 & 11110110 \\
\hline 0111110011 & 01110101 & 1111111101 & 11110111 \\
\hline 0111001100 & 01110110 & 0011111111 & 11111000 \\
\hline 0111111101 & 01110111 & 0000111111 & 11111010 \\
\hline 0001111111 & 01111000 & 0011110011 & 11111011 \\
\hline 1100111100 & 01111010 & 0011001111 & 11111100 \\
\hline 0011111100 & 01111011 & 0000110011 & 11111101 \\
\hline 0001001111 & 01111100 & 0011001100 & 11111110 \\
\hline 0000001111 & 01111101 & 1111111111 & 11111111 \\
\hline 0001001100 & 01111110 & & \\
\hline
\end{tabular}

This mapping, obtained using a strategy similar to the previous one, can be produced using the following boolean equations:

$$
\begin{aligned}
& x_{0}=y_{1} b_{2}, x_{1}=\left(\overline{b_{0}}+b_{5}\right) \overline{b_{6}} \overline{b_{1}} b_{2}+\overline{b_{3}}+x_{0} \\
& x_{2}=z_{2} b_{3} b_{2}, x_{3}=\left(b_{0} z_{0}+z_{2}\right) \overline{b_{7}} y_{9} \\
& x_{4}=y_{2} \overline{b_{5}}+z_{1}+y_{1}, x_{5}=y_{3} \overline{b_{6}} \overline{b_{5}}+y_{6} b_{2} b_{6}+y_{5} y_{9} \\
& x_{6}=\left(y_{8}+b_{1} \overline{b_{4}}\right) b_{2}+y_{8} \overline{b_{4}} b_{3}+y_{7}+b_{0} z_{1}+y_{1} \\
& x_{7}=\overline{b_{0}} \overline{b_{2}}\left(\overline{b_{1}}+b_{3}\right)+y_{2} b_{5}, x_{8}=\left(\overline{b_{7}}+\overline{y_{9}}\right) y_{1}+y_{7} \\
& x_{9}=y_{6} \overline{b_{2}}+y_{4} \overline{b_{3}}+x_{2} b_{6} b_{4}+y_{5}+y_{3} \overline{b_{7}} b_{6} \\
& y_{0}=\overline{b_{1}} \overline{y_{9}}+b_{7} z_{0}+y_{5}+b_{1} y_{9} b_{7}, y_{1}=b_{0} b_{1} \\
& y_{2}=\overline{y_{4}}\left(b_{0} \oplus b_{1}\right) \overline{b_{3}} \overline{b_{2}}, y_{3}=b_{0} \overline{b_{1}} b_{3} \\
& y_{4}=\overline{b_{0}+\overline{b_{4}}}, y_{5}=\overline{b_{0}} \overline{b_{1}}, y_{6}=\overline{b_{0}} b_{3} \\
& y_{7}=x_{2} \overline{b_{6}}, y_{8}=b_{0} b_{7} b_{6}, y_{9}=\overline{\overline{b_{3}}}+b_{2} \\
& z_{0}=\overline{b_{6}} \overline{b_{1}} b_{5}, z_{1}=\overline{b_{3}} b_{2}, z_{2}=\overline{b_{0}} b_{1} \\
& t_{0}=x_{0}+y_{0}, t_{1}=b_{4} y_{0}+b_{3} x_{0}, t_{2}=x_{8}+x_{9}, t_{3}=b_{5} x_{9}+b_{4} x_{8} \\
& t_{4}=x_{6}+x_{7}, t_{5}=b_{6} x_{7}+b_{5} x_{6}, t_{6}=x_{4}+x_{5}, t_{7}=b_{7} x_{5}+b_{6} x_{4} \\
& t_{8}=x_{1}+x_{3}, t_{9}=b_{4} x_{3}+b_{7} x_{1}
\end{aligned}
$$

These equations lead to a circuit of 85 elementary gates $\left(98500 \lambda^{2}\right)$ using Alliance. Synthesis for STMicro $28 \mathrm{~nm}$ FDSOI produces an area of $38.51 \mu \mathrm{m}^{2}$ (62 gates instantiated), and a propagation time of 120 ps.

\section{Technology mapping optimization}

As can be seen in the previous assignment tables, some ternary codes are generated by 2 binary codes because of the "don't cares" ( 5 ternary codes for our first decoder and 13 for the second one). There are potential boolean simplification and technology mapping optimization opportunities left by assigning only one binary code per ternary code and specifying a "don't care" output for the unused binary codes. However, there is no general optimization pattern that we could find to select one of these two possibilities for the 5 (respectively 13) cases so as to minimize the number of gates resulting from the implementation. As the number of possible combinations is $2^{5}=32$ for the first decoder and $2^{13}=8192$ for the second one, we decided to use a brute-force approach. Indeed, these numbers are small enough that we can synthesize all theses cases in a few days ${ }^{1}$. For all synthesized circuits, we plot the critical path as a function of the area in Figures 1 and 2. The size of the dot is proportional to the number of cases that match a given (area, time).

${ }^{1}$ Synthesizing all $5 \mathrm{~b}$-to-3t cases on a medium range server actually took a bit more than 4 hours, and all $8 \mathrm{~b}-$ to-5t cases two and a half days. 


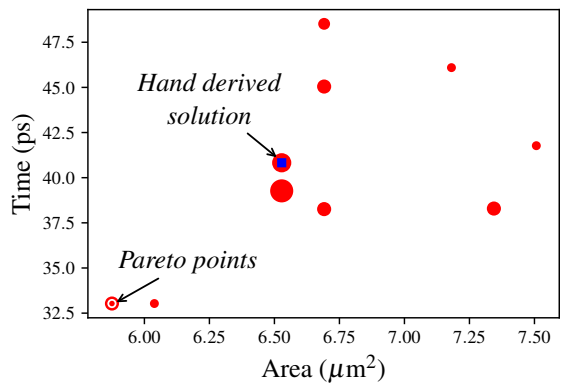

Fig. 1: Design space for the 5 bits to 3 trits decoder

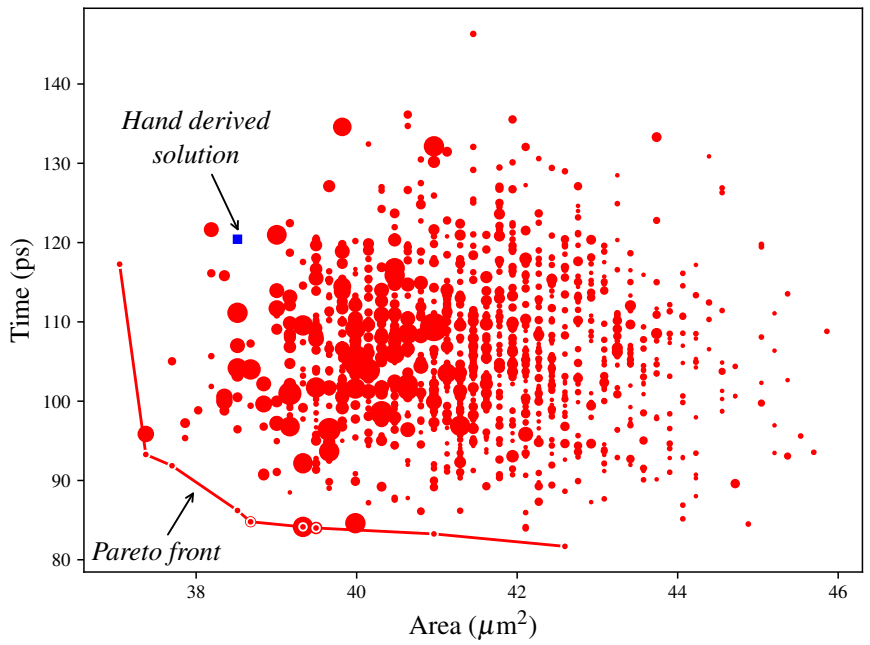

Fig. 2: Design space for the 8 bits to 5 trits decoder

As expected, there are better solutions than our original hand-derived one, for area and/or time. However, some of them are worse for both area and time, even though they actually are just subsets of our initial designs. The performance of the optimization process of the synthesis tool (and maybe the targeted technology) is then key for the quality of the solutions and none can be said to be the best in all conditions, as the Pareto front in the figures shows. The search and the choice of the best trade-off has to be done by the users of the decoder with their tool, technology, and target applications.

\section{Encoding}

For completeness, we also now give the number of gates, area and propagation time for the encoding part of our decoders. If needed, the equations of the encoder can be generated by a boolean minimization program (e.g. Espresso) using the reverse table as entry point. Please note that since we are focused on reading and decompressing the code, the values written into memory may well be computed offline by software instead of requiring dedicated encoding hardware.

1) 3 trits to 5 bits: Using the reversed version of the mapping of subsection V-A using Synopsys on STMicro $28 \mathrm{~nm}$ FDSOI gives an area of $18.44 \mu^{2}$ (29 gates instantiated), and a propagation time of 92 ps.

2) 5 trits to 8 bits: Identically, for the mapping of subsection V-B, we obtain an area of $102.7 \mu^{2}$ (172 gates instantiated), and a propagation time of 178 ps using STMicro $28 \mathrm{~nm}$ FDSOI technology.

\section{AREA SAVINGS}

As illustrated in Figure 3, the area overhead brought by our decoders depends only on the memory width (in bits) and not on the memory depth (number of words). So no matter how large the decoder, there will always exist a minimum number of words above which the memory area savings are higher than the decoder overhead.

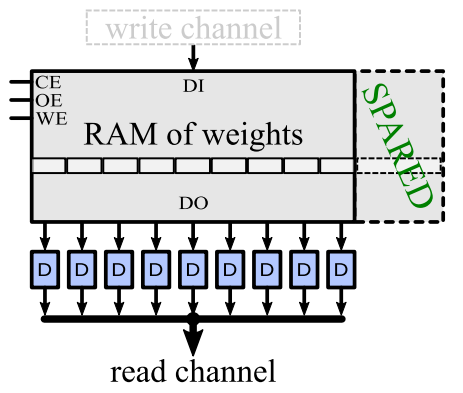

Fig. 3: Impact of decoding (D) on an ternary ANN weight memory. Weights are written once at configuration time, and read simultaneously during inference.

In $[20]^{2}$, the authors report an SRAM bit cell area of $A_{B}=$ $0.12 \mu \mathrm{m}^{2}$ in the technology we use, also for an ANN application. Given this information, we can derive rough but credible estimates of the size of a memory cut of $W$ words of $B$ bits each, and decide when it is interesting to use our encoding approach. We note $D$ the number of decoders and $A_{D}$ the area of one decoder. For the 3-trit case, we have $D=\frac{B}{6}$. The area overhead of the decoders is $A_{D} \times D=A_{D} \times \frac{B}{6}$. The memory area spared is $D \times W \times A_{B}=\frac{B}{6} \times W \times A_{B}$. Hence, to obtain a saving of $R$ as ratio of the original memory size, the condition is:

$$
\frac{B}{6} \times W \times A_{B}-A_{D} \times \frac{B}{6}>R \times W \times B \times A_{B}
$$

Which simplifies to:

$$
W>\frac{A_{D}}{A_{B}} \times \frac{1}{1-R \times 6}
$$

A similar argument gives in the 5-trit case:

$$
W>\frac{A_{D}}{A_{B}} \times \frac{1}{2-R \times 10}
$$

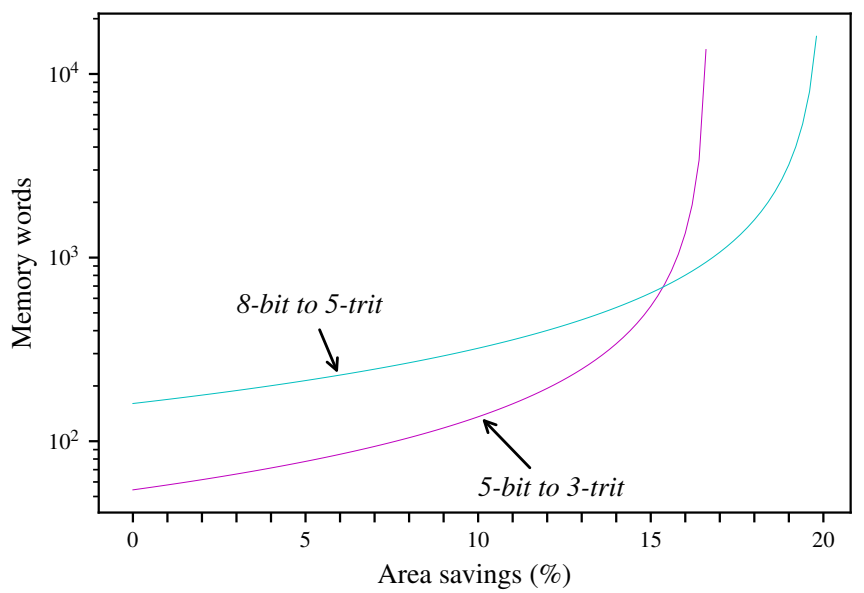

Fig. 4: Minimum memory size for a given area savings

Figure 4 gives the value of $W$ such that Equation 2 (3-trit case) and 3 (5-trit case) hold for continuous values of $R$. It shows that the 3-trit approach is more interesting for small memories, while the highest area

\footnotetext{
${ }^{2}$ Slide 25 of their oral ISSCC presentation.
} 
savings are obtained with the 5-trit approach. At the intersection point, both approaches bring overall savings of $15.4 \%$ with 691 memory words. The need for memories of such size is very common in the context of neural network architectures. For example in the AlexNet network [21], the largest layers feature 4096 neurons with 4096 weight values per neuron. The corresponding memories of weights, both deep and wide, are perfect candidates to ternary compression. The proposed compression approaches, completely devoid of control, are also among the few-if not the only ones-suitable for such wide memories under a sustained throughput measured in $\mathrm{Tb} / \mathrm{s}$.

Clearly, any better trits-to-bits mapping associated to an optimized logic synthesis process would enable lowering these thresholds, hence the interest in any approach that could address this class of problems.

\section{POWER CONSIDERATIONS}

SRAM memories are known to consume orders of magnitude more than logic gates [22], even with relatively small memories. This is still the case with the technology we use, although the raw data is not publicly accessible. The power - and area — of memory cuts depends a lot on technological features and architectural-level parameters anyway, so we provide power results as a general trend only.

We did power simulations with the STMicro $28 \mathrm{~nm}$ FDSOI technology, assuming a toggle rate of $50 \%$ on the inputs (standard assumption, but high in the context of ternary ANN where zero weights dominate). We observed that the 5-trit decoder consumes roughly $8 \times$ more than the 3 -trit decoder. However, due to the much higher consumption of the SRAM, the 5-trit approach brings overall better power savings than the 3 -trit one thanks to its better compression ratio ( $-20 \%$ versus $-16.7 \%$ in memory width). Both decoding solutions bring around $15 \%$ power savings for small memories, e.g. 512 words, with a slight advantage for the 5 -trit approach. Higher savings, closer to the $20 \%$ limit, can be obtained with the 5 -trit approach for deeper and/or wider memories, e.g. around $18 \%$ overall power savings are observed with $4 \mathrm{k}$ words.

In the case of external DRAM access, the power consumption of decoding (and even encoding) is so insignificant compared to DRAM operations that the proposed approaches would bring a solid $16.6 \%$ power savings for the 3-trit approach and $20 \%$ savings for the 5-trit, along with similar reduction in memory size requirements.

\section{SUMMARY}

In this correspondence, we address the problem of efficiently decompressing a vector of bits into binary encoded trits. We first show that it is neither necessary nor practical to compress more than 5 trits into 8 bits, and then give two optimized mappings and their corresponding multivalued and multilevel boolean function. These mappings were obtained by human reasoning, and no automatic method we could think of gave better or even approaching results. It is left as an open problem to know if better mappings exist.

In conclusion, the proposed approaches bring noticeable savings both on area and power, which makes them essential in all classes of applications where ternary values are stored in memory and read frequently.

\section{ACKNOWLEDGMENTS}

This work is funded in part by Grenoble Alpes Métropole through the Nano2017 Esprit project. We want to acknowledge CMP for the provision of products and services that facilitated this research, including access to STMicroelectronics $28 \mathrm{~nm}$ design kits and macroblock generators data. Finally, we would like to thank Olivier Menut (STMicroelectronics, Crolles) for insightful discussions during the project and Mario Diaz-Nava (ST Microelectronics, Grenoble) for his long-term encouragement and support.

\section{REFERENCES}

[1] N. P. Brusentsov and J. R. Alvarez, "Ternary computers: The setun and the setun 70," in Perspectives on Soviet and Russian Computing. Springer, 2011, pp. 74-80.

[2] Z. Sahinoglu and S. Gezici, "Ranging in the ieee 802.15. 4a standard," in IEEE Annual Wireless and Microwave Technology Conference, 2006. IEEE, 2006, pp. 1-5.

[3] M. Abdelaziz and T. A. Gulliver, "Ternary convolutional codes for ternary phase shift keying," IEEE Communications Letters, vol. 20, no. 9, pp. $1709-1712,2016$

[4] J. Nystad, A. Lassen, A. Pomianowski, S. Ellis, and T. Olson, "Adaptive scalable texture compression," in Proceedings of the Fourth ACM SIGGRAPH/Eurographics conference on High-Performance Graphics. Eurographics Association, 2012, pp. 105-114.

[5] A. V. Burlakov, M. V. Chekhova, O. A. Karabutova, D. N. Klyshko, and S. P. Kulik, "Polarization state of a biphoton: Quantum ternary logic," Physical Review A, vol. 60, no. 6, p. R4209, 1999.

[6] A. K. Ghosh and A. Basuray, "Binary to modified trinary number system conversion and vice-versa for optical super computing," Natural Computing, vol. 9, no. 4, pp. 917-934, 2010.

[7] F. Li, B. Zhang, and B. Liu, "Ternary weight networks," arXiv preprint arXiv:1605.04711, 2016.

[8] A. Prost-Boucle, A. Bourge, F. Pétrot, H. Alemdar, N. Caldwell, and V. Leroy, "Scalable high-performance architecture for convolutional ternary neural networks on fpga," in 27th International Conference on Field Programmable Logic and Applications. IEEE, 2017, pp. 1-7.

[9] A. Prost-Boucle, A. Bourge, and F. Pétrot, "High-efficiency convolutional ternary neural networks with custom adder trees and weight compression," ACM Trans. Reconfigurable Technol. Syst., vol. 11, no. 3, pp. 15:1-15:24, Dec. 2018.

[10] R. K. Brayton, G. D. Hachtel, and A. L. Sangiovanni-Vincentelli, "Multilevel logic synthesis," Proceedings of the IEEE, vol. 78, no. 2, pp. 264-300, 1990

[11] K. A. Bartlett, R. K. Brayton, G. D. Hachtel, R. M. Jacoby, C. R. Morrison, R. L. Rudell, A. Sangiovanni-Vincentelli, and A. Wang, "Multi-level logic minimization using implicit don't cares," IEEE Transactions on Computer-Aided Design of Integrated Circuits and Systems, vol. 7, no. 6, pp. $723-740,1988$.

[12] S. Devadas and A. R. Newton, "Exact algorithms for output encoding, state assignment, and four-level boolean minimization," IEEE Transactions on Computer-Aided Design of Integrated Circuits and Systems, vol. 10, no. 1, pp. 13-27, Jan. 1991.

[13] G. De Micheli, R. K. Brayton, and A. Sangiovanni-Vincentelli, "Optimal state assignment for finite state machines," IEEE Transactions on Computer-Aided Design of Integrated Circuits and Systems, vol. 4, no. 3, pp. 269-285, 1985.

[14] G. De Micheli, Synthesis and optimization of digital circuits. McGrawHill Higher Education, 1994.

[15] S. Mitra, L. J. Avra, and E. J. McCluskey, "An output encoding problem and a solution technique," IEEE Transactions on Computer-Aided Design of Integrated Circuits and Systems, vol. 18, no. 6, pp. 761-768, Jun. 1999.

[16] L. Burgun, N. Dictus, A. Greiner, E. P. Lopes, and C. Sarwary, "Multilevel logic optimization of very high complexity circuits," in Proceedings of the European Design Automation conference. IEEE Computer Society Press, 1994, pp. 14-19.

[17] P. C. McGeer, J. V. Sanghavi, R. K. Brayton, and A. SangiovanniVicentelli, "Espresso-signature: A new exact minimizer for logic functions," IEEE Transactions on Very Large Scale Integration Systems, vol. 1, no. 4, pp. 432-440, 1993.

[18] H. W. Kuhn, "The hungarian method for the assignment problem," Naval Research Logistics Quarterly, vol. 2, pp. 83-97, 1955.

[19] E. J. McCluskey Jr, "Minimization of boolean functions," Bell system technical Journal, vol. 35, no. 6, pp. 1417-1444, 1956.

[20] G. Desoli, N. Chawla, T. Boesch, S. p. Singh, E. Guidetti, F. D. Ambroggi, T. Majo, P. Zambotti, M. Ayodhyawasi, H. Singh, and N. Aggarwal, "A 2.9tops/w deep convolutional neural network soc in fd-soi $28 \mathrm{~nm}$ for intelligent embedded systems," in 2017 IEEE International Solid-State Circuits Conference, Feb 2017, pp. 238-239.

[21] A. Krizhevsky, I. Sutskever, and G. E. Hinton, "Imagenet classification with deep convolutional neural networks," in Advances in neural information processing systems, 2012, pp. 1097-1105.

[22] A. Pedram, S. Richardson, M. Horowitz, S. Galal, and S. Kvatinsky, "Dark memory and accelerator-rich system optimization in the dark silicon era," IEEE Design \& Test, vol. 34, no. 2, pp. 39-50, 2017. 